\title{
Deconstruyendo el modelo deficitario de la apropiación social de la ciencia y la tecnología en Colombia: el caso de la cartilla "Las Maticas de mi Huerta"
}

\section{Desconstruindo o modelo de déficit na apropriação social da ciência e tecnologia na Colômbia: o caso da cartilha "Las Maticas de mi Huerta"}

Deconstructing the deficit model of social appropriation of science and technology in Colombia: the case of the handbook "Las Maticas de mi Huerta"

\author{
Marcela Lozano-Borda ${ }^{2}$ \\ Tania Pérez-Bustos ${ }^{3}$ \\ Carolina Roatta-Acevedo ${ }^{4}$
}

\begin{abstract}
${ }^{1}$ Este artículo se enmarca en el componente de análisis de materiales del proyecto Centro de Innovación y Recursos para la Apropiación Social de la Ciencia y la Tecnología de la Corporación Maloka y Colciencias. El orden de las autoras es alfabético y no representa una jerarquía.

${ }^{2}$ Comunicadora Social, Máster en Comunicación Científica, Médica y Medioambiental. Investigadora del Observatorio Colombiano de Ciencia y Tecnología, Docente de Cátedra Facultad de Comunicación y Lenguaje, Pontificia Universidad Javeriana. E-mail: mlozanob@ocyt.org.co.

${ }^{3}$ Antropóloga y Comunicadora Social, Maestría en Estudios del Desarrollo y Doctorado en Educación, Profesora-Investigadora Facultad de Ciencias Sociales, Pontificia Universidad Javeriana. Profesora Invitada de la Escuela de Estudios de Género de la Universidad Nacional de Colombia. Investigadora independiente: Maloka. E-mail: tpbustos@gmail.com.

${ }^{4}$ Comunicadora Social - Periodista, candidata a Maestría en Estudios Culturales de la Universidad Nacional de Colombia. Investigadora junior Corporación Maloka, Docente de Cátedra Departamento de Comunicación Social - Periodismo, Universidad Central. E-mail: caroroatta@gmail.com.
\end{abstract}




\title{
RESUMEN
}

Los estudios sobre política y actividades de apropiación social de ciencia y tecnología (ASCyT) en Colombia han evidenciado la presencia de un modelo deficitario de relación entre el conocimiento científico y el público. Este modelo, que está presente en diversidad de actividades educomunicativas, se caracteriza por describir dinámicas de comunicación unidireccional y vertical de la ciencia hacia sus públicos. Estos estudios se han quedado cortos en identificar cuáles son las condiciones de producción que sostienen dicho modelo. Analizando el caso de una cartilla educativa, este artículo busca profundizar en la comprensión de aquello que define la ASCyT en Colombia como una práctica deficitaria. Lo anterior se logró desde un análisis de contenido etnográfico de este material educativo que permitió identificar una serie de dispositivos de lenguaje y gráficos que pueden a futuro servir de guía reflexiva para el diseño de otros materiales edu-comunicativos para la ASCyT.

Palabras-clave: apropiación social de la ciencia y la tecnología; materiales edu-comunicativos; modelo deficitario entre ciencia-público.

\section{RESUMO}

Estudos sobre políticas e atividades de apropriação social da ciência e tecnologia (ASCT) na Colômbia têm mostrado a presença de um modelo de déficit de relação entre o conhecimento científico e o público. Este modelo, que está presente em diversas atividades educacionais e comunicativas, se caracteriza por estabelecer uma dinâmica de comunicação unidirecional e vertical da ciência para seus públicos. Contudo, estes estudos não têm identificado quais são as condições que sustentam este modelo de produção. Analisando o caso de uma cartilha educativa, este artigo procura aprofundar a compreensão sobre o que define a prática da ASCT na Colômbia como deficitária. Isto foi realizado a partir de uma análise de conteúdo etnográfico do material, a partir do qual foi possível identificar uma série de dispositivos de linguagem e de diagramação que no futuro podem servir como guia reflexiva para o desenho de novos materiais educomunicativos para a ASCT.

Palavras-chave: apropriação social da ciência e da tecnologia; materiais educomunicativos; modelo de déficit entre ciência-público.

\begin{abstract}
Studies on policy and on activities of social appropriation of science and technology (SAST) in Colombia have shown the presence of a deficit model that characterizes the relationship between scientific knowledge
\end{abstract}


and the public. This model, which is present in diverse communicative and educational activities, is characterized by establishing unidirectional and vertical communication dynamics from science to its public. However, these studies have fallen short in identifying what the production conditions that sustain this model are. Analyzing the case of an educational booklet, this article seeks to deepen the understanding of what it is that defines the SAST practice in Colombia as deficitarian. This was achieved through an ethnographic content analysis of educational material, which allowed the identification of a number of language and graphic devices that, in the future, could become a reflexive guide in the design of new educational and communicative materials for the SAST.

Keywords: social appropriation of science and technology; edu-comunicative materials; deficit model of science-public.

\section{Introducción}

Los estudios sobre política y actividades de apropiación social de ciencia y tecnología (ASCyT) en Colombia ${ }^{5}$ han evidenciado la presencia de un modelo deficitario de comunicación que define una cierta relación y jerarquías entre el conocimiento científico y el público al que se dirigen, que se caracteriza por el establecimiento de una relación unidireccional y vertical de la ciencia al público (DAZA; ARBOLEDA, 2007; PÉREZ-BUSTOS, 2010b; PÉREZ-BUSTOS et al., 2012; FRANCO-AVELLANEDA; PÉREZ-BUSTOS, 2010). Este modelo deficitario tiene dos características principales. En primer lugar, la estandarización en países como Colombia de modelos educativos utilizados en dichas actividades, a partir de propuestas de popularización y divulgación desarrolladas en Estados Unidos y Europa. Esto refuerza una segunda característica, la instrumentalización del papel de quienes median comunicativa y educativamente estos modelos, que en el marco de lógicas de mercadeo y consumo, contribuyen a despolitizar el acto educativo en ciencia y tecnología (PÉREZ-BUSTOS, 2010a, 2010 b, 2010c). Junto con esto, las actividades enmarcadas en estos modelos privilegian la experimentación como forma de conocimiento empírico - inductivo (FRANCO-AVELLANEDA; PÉREZ-BUSTOS, 2009), promoviendo un

${ }^{5}$ La noción de apropiación social del conocimiento se ha ido acotando en Colombia y algunos países de América Latina para describir en la formulación de políticas, el conjunto de actividades que en otros contextos son conocidas como popularización científica, divulgación científica o comunicación pública de la ciencia y la tecnología. (FRANCO-AVELLANEDA; PÉREZ-BUSTOS, 2010). 
ideal de ciencia ilustrada neutra, libre de valores, moralmente incuestionable, símbolo de la superioridad cultural de los países occidentales sobre las antiguas colonias $^{6}$. Otra característica de estos modelos es que presentan a los mediadores como transmisores del conocimiento científico y a los públicos como sujetos infantilizados ${ }^{7}$, ambos en una relación de subordinación frente a ese ideal de ciencia como saber legítimo.

Al rastrear los "cómo" de las actividades de ASCyT (PÉREZ-BUSTOS et al., 2012), encontramos que estas dinámicas de estandarización en Colombia se relacionan con una tendencia a homologar la ASCyT con la producción de materiales edu-comunicativos como cartillas, folletos, libros, etc., no sólo para las instituciones que se caracterizan por ser mediadoras de estas prácticas como los museos, centros interactivos y ferias de ciencia, sino también para otros actores que están promoviendo la ASCyT, como las empresas, el Estado, la comunidad científica y la sociedad civil. Existe igualmente, una tendencia a asumir la participación de los públicos desde la información, donde este ejercicio comunicativo parte de un supuesto del público como receptor pasivo de un mensaje, lo que lo coloca en un lugar subordinado frente al conocimiento científico.

Con miras a comprender las formas en que se materializa este modelo de relación entre ciencia y público, el cual circula principalmente en materiales editoriales, presentamos en este artículo un análisis de contenido etnográfico sobre la cartilla Las Maticas de mi Huerta, dirigida a población campesina de los sectores rurales de Bogotá, capital de Colombia y producida en 2007 por el Jardín Botánico "José Celestino Mutis" y la Secretaría Distrital de Ambiente de esta ciudad. Esta fue creada con el objetivo de socializar con la comunidad que participó en la investigación etnobotánica, los resultados del proyecto de "Uso Sostenible de los Recursos Vegetales del Distrito Capital y la Región" llevado a cabo por la Subdirección Científica del Jardín Botánico en varias localidades de Bogotá que cuentan con población campesina. La publicación también hizo parte del trabajo de grado de las autoras para obtener el título de Licenciatura en Biología de la Universidad Pedagógica Nacional.

Así, presentaremos en una primera parte algunas precisiones teórico-metodológicas acerca del trabajo realizado, seguida por un segundo apartado

\footnotetext{
${ }^{6}$ Esto dialoga con los planteamientos de Nieto (2000) y Castro Gómez (2005) sobre la colonialidad.

${ }^{7}$ La idea de menor de edad es planteada por Kant (1783) en su ensayo sobre "Respuesta a la pregunta: ¿qué es la ilustración”. Kant fue uno de los padres de la antropología, la cual fue creada inicialmente como una "sociología de los pueblos primitivos". Para el caso de este artículo nos interesa subrayar que esta noción de "infantilizado" de "menor de edad" que no puede tomar decisiones tecnocientíficas por si mismo hace que la ASCyT se asocie con la función social de las mujeres de crianza y cuidado (PÉREZ-BUSTOS, 2010a).
} 
con los resultados del análisis que nos permitió identificar algunos dispositivos de circulación del modelo deficitario.

\section{Algunas precisiones teórico-metodológicas}

Entendemos la comunicación como producción de discurso entre interlocutores que comparten códigos y referentes, bajo condiciones históricas, culturales e ideológicas (HALL, 1997; ORLANDI, 2009; GALIETA NASCIMENTO; CASSIANI, 2009; CASSIANI; LINSINGEN; GIRALDI, 2011). En el caso de la cartilla, quisimos analizar los recursos de lenguaje y diagramación que se configuran en ella y que contribuyen a posicionar y circular un modelo deficitario de relación entre ciencia y públicos y las condiciones de producción de ésta. Si bien en la investigación más amplia ya hemos iniciado con las autoras de estos materiales y con otros mediadores que también diseñan productos edu-comunicativos, un proceso de discusión sobre las condiciones de producción de estos recursos de lenguaje y diagramación para este artículo nos interesa profundizar en caracterizarlos en la materialidad de la cartilla en cuestión.

El ejercicio analítico realizado se apoya en una interpretación etnográfica del contenido de la cartilla, abordando preguntas como: ¿de qué manera se exponen los conocimientos que se quieren comunicar?, ¿a través de qué dispositivos del lenguaje?, ¿quiénes lo hacen?, ¿cómo se representan gráficamente? En relación con esto, nos interesó entender qué representaciones sobre el conocimiento científico estaban puestas en escena en este material y qué tipo de relaciones ellas establecían con un sujeto lector, en genérico, el público.

La investigación que aquí presentamos ha sido una oportunidad para pensar en nuestro lugar de mediadoras del conocimiento científico, dado que hemos tenido la experiencia de diseñar actividades que promueven la ASCyT, entre ellas la producción de materiales editoriales ${ }^{8}$. Reconocemos que este trabajo está inserto en una lógica particular de la ASCyT cuyas prácticas edu-comunicativas

${ }^{8}$ Algunos ejemplos de ello son: una publicación seriada de Maloka para docentes de educación básica y media denominada "Periódico Encuentro". Una cartilla para jóvenes de educación media llamada "Aire" realizada con apoyo de la Secretaría Distrital de Ambiente. Una colección editorial sobre temas de Energía, Matemáticas, Agua de apoyo a las exposiciones interactivas de Maloka. Está igualmente la redacción de contenidos para las exposiciones interactivas de "Sala de Telecomunicaciones", "Monstruos Marinos" y "La Aventura de Construir Nación: Historia de la Ciencia y la Tecnología en Colombia" de este mismo Centro Interactivo. 
están al servicio de una cierta idea hegemónica de conocimiento científico neutral, bueno, loable y a la actividad de sus expertos ${ }^{9}$. Desde allí proponemos un ejercicio de deconstrucción de un material edu-comunicativo en particular que a futuro permita pensar nuevas formas de hacer apropiación que cuestionen el modelo deficitario que se da entre expertos, mediadores y públicos.

En esa línea, planteamos un análisis de contenido etnográfico de la cartilla (ALTHEIDE, 1987) en busca de los mecanismos que se usan y que ratifican la existencia del modelo deficitario ya mencionado. A partir de esta revisión agrupamos tendencias que nos permitieron identificar ciertos dispositivos de lenguaje y gráficos. Éstos fueron descritos en el contexto de la cartilla, permitiendo percibir lo manifiesto, lo directo, lo que las autoras pretendían comunicar a través de la cartilla; por supuesto, identificando no solo lo explícitamente dicho, sino aquel texto oculto, latente, indirecto. Esto nos permite encontrar sentidos o significados múltiples en función de la perspectiva y el punto de vista de quien los lee, los cuales pueden ser diferentes al sentido que pretendían darle las autoras originales.

\section{Resultados y análisis}

Como ya señalamos, la cartilla Las Maticas de mi Huerta fue producida por el Jardín Botánico de Bogotá con la financiación de la Secretaría Distrital de Ambiente en el año 2007, en el marco del proyecto 318 "Uso Sostenible de los recursos vegetales del distrito capital y la región”. Está dirigida a público campesino, en especial a quienes participaron en el desarrollo del proyecto y su objetivo es:

Brindar información práctica en forma clara con un lenguaje coloquial que la hace alegre y entretenida. Esperamos que contribuya a la recuperación

\footnotetext{
${ }^{9}$ Pérez-Bustos (2010a) ha descrito esta práctica como feminizada. Con ello ha subrayado, que como con la educación básica y media, el hecho de que estas prácticas estén principalmente siendo promovidas por mujeres, es un síntoma de un fenómeno cultural que asocia la tarea educativa, en este caso de educar en ciencia y tecnología, con una práctica de cuidado de sujetos infantilizados. Esta asociación simbólica con una cierta idea de lo femenino también implica que estas prácticas, simbólicamente, se configuran al servicio de una cierta idea de ciencia que es vista por la crítica feminista como androcéntrica, en tanto que reproduce unos ideales de neutralidad y modestia propios de la masculinidad de los científicos europeos del siglo XVII.
} 
de nuestros recursos vegetales y a su justa valoración; así mismo, que se constituya en material de consulta permanente por todos los miembros de la familia y en un apoyo importante en las escuelas cuando se traten temas de botánica y de ciencias naturales. (JBJCM, 2007, p. 2)

La primera parte de la cartilla está compuesta por algunas narraciones del personaje principal, el campesino Cándido Buendía. Este actor inicia contando una historia sobre su abuela, señalando que es importante rescatar el conocimiento que ella tiene acerca de las plantas de la región, sus formas de cultivo, su cuidado y sus usos; ese saber que ha venido de generación en generación. Tras ello, Cándido, siempre sonriente en las ilustraciones del material, le dice al lector que compartirá estos saberes, sin olvidar lo que dicen "los estudiosos", sobre las plantas de la región (ver imagen 1). Sin embargo, como señalaremos más adelante, los conocimientos de la abuela no aparecerán con la misma fuerza que los de los expertos, los "estudiosos" a los que se refiere en la apertura de este material edu-comunicativo.

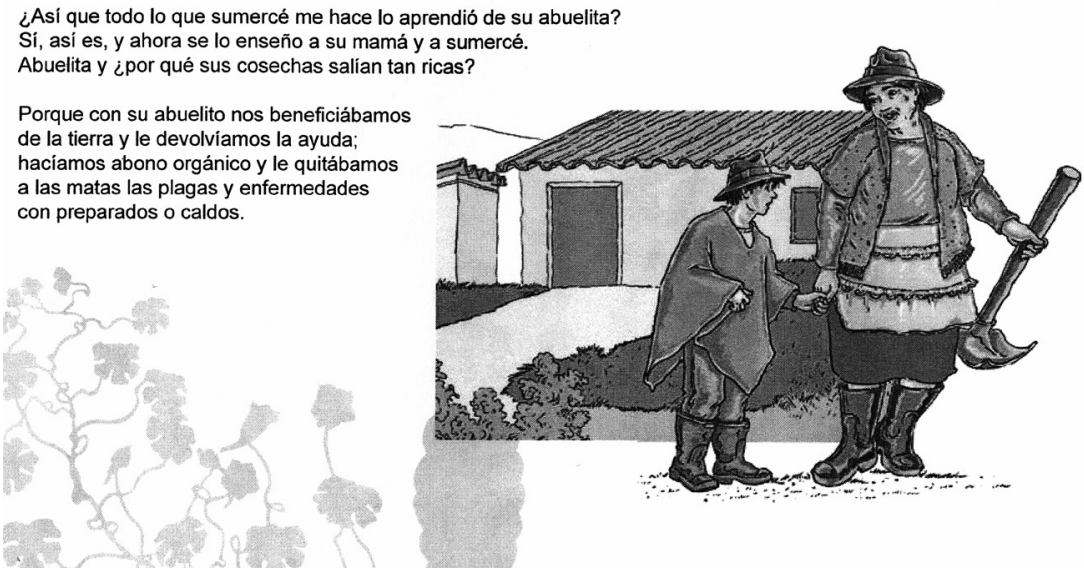

Imagen 1 - Fonte: Cartilla Las Maticas de mi Huerta. Jardín Botánico José Celestino Mutis, Bogotá.

En la segunda parte conformada por tres capítulos, podemos encontrar la descripción taxonómica de varias plantas de la región altoandina ${ }^{10}$, cómo hacer su siembra apropiadamente y cuáles son los cuidados que deben tenerse con

${ }^{10}$ Esa denominación está haciendo alusión a ecosistemas que se encuentran en las partes superiores de las cordilleras andinas de Colombia. 
estos cultivos. En este apartado predomina el conocimiento experto, mientras el conocimiento del campesino y sus ancestros queda relegado al nivel de anécdotas y datos curiosos que más que un complemento importante, se utilizan como un recurso narrativo accesorio y en muchos casos meramente decorativo.

En la última parte del material, encontramos un recetario de conservas, yogur y harinas que se pueden elaborar con las plantas anteriormente descritas. Para facilitar la tarea de la preparación de las harinas, el material propone la construcción de un secador solar de manera didáctica: describiendo los elementos y procedimientos en detalle, a través de gráficos. En este apartado, al igual que en el anterior, el conocimiento de la abuela de Cándido aparece representado a través de frases y expresiones más de tipo "accesorio". Es notorio que si sus comentarios desaparecen, el texto no pierde unidad ni coherencia.

La cartilla cierra con una breve despedida de Cándido y con la letra de una canción de Jorge Velosa. ${ }^{11}$ El uso de este recurso apela a la inclusión de aspectos del mundo cultural de un público campesino estereotipado, sin embargo, como lo analizaremos más adelante, estos textos se relacionan "decorativamente" con los demás apartados de la cartilla, banalizando la relación con la expresión popular (PÉREZ-BUSTOS, 2010c).

\section{Las dos voces de la cartilla}

Hemos mencionado que la ciencia y la tecnología están entramadas por relaciones de poder que han subordinado otras formas de conocer. Es común encontrar prácticas de comunicación científica enmarcadas en modelos deficitarios en los que el ideal de sujeto que las ejerce está definido por un tipo de ciencia y tecnología dominante, el cual reproduce unos estereotipos de ciencia en los que se privilegia una idea de conocimiento dada, neutral, circunscrita a ciertos escenarios de producción de saber en particular. Esta subordinación que privilegia un modelo vertical y unidireccional de comunicación, se representa en la cartilla analizada, a través de dos voces narrativas: la de un campesino y la de un experto. El material intenta ofrecer la perspectiva del campesino, desde su cotidianidad y expresión, sin embargo, ello se deslegitima cuando se convoca al experto como autoridad, como voz ausente pero fuertemente presente cuando

${ }^{11}$ Cantautor colombiano que adquirió fama por ser el co-iniciador del género musical conocido como carranga. Esta música campesina, corresponde a un género de música folclórica surgida en la región andina colombiana. 
solo su saber es el que finalmente tiene validez. El campesino se constituye en un recurso instrumental y accesorio subordinado que legitima la voz del experto. En contraposición, el experto presenta la noción de un conocimiento científico certero, ordenado, preciso e inmutable.

\section{Los recursos de lenguaje y diagramación como dispositivos}

Lo anteriormente mencionado lo precisaremos en el análisis que exponemos a continuación. En nuestro análisis etnográfico del material (ALTHEIDE, 1987) emergieron unas categorías relacionadas con los dispositivos del lenguaje y gráficos identificados en el material. Este análisis partió de la hipótesis de que la cartilla reproduce en sus representaciones de públicos y expertos el modelo deficitario que ha caracterizado las prácticas de comunicación de la ciencia y la tecnología en el país (PÉREZ-BUSTOS et al., 2012).

Identificamos como dispositivos del lenguaje: la personalización, las definiciones, las marcas de informalidad o formalidad y la narración; y como dispositivos gráficos analizamos el papel de: las ilustraciones, las fotografías, las tablas y la diagramación. La descripción general de cada uno de estos componentes la exponemos a continuación.

\section{Dispositivos del lenguaje}

Personalización: con este dispositivo se espera que el lector encuentre actitudes, valores y opiniones de una persona parecida a él. Es decir, que se presenta y describe a un personaje en el material que tiene la capacidad de representar al lector mismo.

Definición: de un lado se utiliza para dar cuenta de un objeto, sus usos y posibilidades, de otro lado, para referirse explícitamente a un significado o término especializado. Este recurso predomina en el discurso científico, en la cartilla veremos que es usado de manera más recurrente por la voz del experto.

Marcas de informalidad o formalidad: se refieren a aquellos términos, expresiones, conceptos, etc. que representan un nivel coloquial o formal en el texto. Es frecuente encontrar en materiales editoriales divulgativos las expresiones coloquiales, que intentan hacer parecer el texto más sencillo de leer por un público no experto. En tanto, los niveles formales en el léxico son más característicos del discurso científico, especializado (BERRUECOS, 2005). En la cartilla encontraremos estos dos tipos de marcas, la primera asociada al campesino y la segunda a la de la voz del experto. 
Narración: narrativizar una explicación es un recurso para acercar un conocimiento especializado a un público no experto, es un dispositivo que comúnmente se ha utilizado en textos divulgativos (CALSAMIGLIA et al., 2001). A través de secuencias narrativas, diálogos y anécdotas de personajes (explícitos), en unos tiempos definidos, se van contextualizando los conceptos y conocimientos científico-tecnológicos, como puede observarse en la cartilla.

\section{Dispositivos gráficos}

Ejemplificación: la inclusión de ejemplos es un mecanismo característico de los textos explicativos (CALSAMIGLIA, TUSÓN, 1999). El uso de ejemplos concretos para dar cuenta de nociones complejas o abstractas se da principalmente en la cartilla analizada a través de las fotografías. Las fotografías presentan primeros planos, detalles de las plantas que refuerzan una idea de precisión y podría decirse que tienen un papel de nivel técnico en la cartilla.

Descripción: uno de los principales recursos utilizados en el material analizado es la terminología específica de disciplinas como la biología y la agronomía, que se presenta de manera ordenada y sistemática. A través de tablas, en la cartilla se exponen los principales términos, nombres científicos y componentes de las plantas enunciadas en el material.

Escenificación: las actitudes y comportamientos de los personajes, así como los escenarios que habitan, se muestran en el material a través de ilustraciones. Por medio de éstas es posible dar cuenta del contexto de la historia que se narra en la cartilla.

Juntos, estos dispositivos nos permiten obtener una mirada general del material, encontrar tendencias y ver de qué manera se da la relación experto campesino a través del mismo.

\section{Los dispositivos como elementos para el análisis}

Siguiendo lo anterior, en el texto podemos encontrar dos voces que exponen los contenidos de la cartilla. Una más cercana y explícita, y otra más distante e implícita. Explícitamente encontramos al campesino Cándido Buendía, que hace la tarea de divulgador, quien traduce introduciendo sus saberes, particularmente en lo que respecta a sus formas de nombrar el mundo. Y de manera implícita, casi invisible porque no hay desde lo gráfico una figura que lo represente, encontramos la voz del experto, que puede identificarse por el tono y léxico utilizado 
y su consecuente "desentonación” con la voz de quien media: Cándido. Estas dos voces se hacen evidentes a través de los dispositivos del lenguaje y gráficos presentes en el material. Veamos la imagen 2 para un ejemplo:

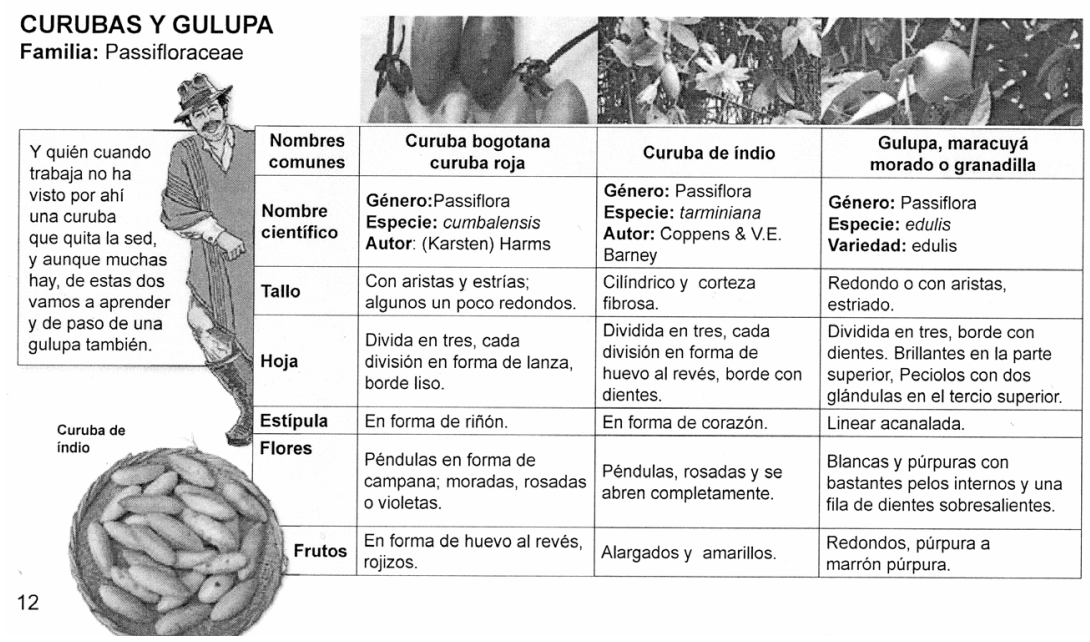

Imagen 2 - Fonte: Cartilla Las Maticas de mi Huerta. Jardín Botánico José Celestino Mutis, Bogotá.

En la imagen encontramos que la presencia del protagonista, Cándido Buendía, es bastante importante, se ubica en el centro de la página y cubre un buen espacio de la misma. Al lado izquierdo de la ilustración podemos observar un recuadro en el que se expone, a manera de viñeta, lo que el campesino dice. Al lado derecho encontramos una tabla explicativa de la planta que se enuncia en el título: la Curuba, con su nombre científico y partes. En los espacios superior e inferior de la tabla vemos fotografías en primer plano de la fruta descrita.

Pese a que la figura de Cándido es muy relevante gráficamente, no es su voz la que valida el conocimiento expuesto en el material, es la voz de un "otro" la que lo hace. Lo curioso es que ese "otro" es invisible, aparentemente no está, pero es su voz la que en realidad predomina en la cartilla, el conocimiento que expone, su intencionalidad de clasificación y ordenamiento es la esencia del texto. Legitimando un tipo de conocimiento occidental, de ordenamiento de la naturaleza que predomina sobre la voz popular aludiendo a formas de colonialidad (NIETO, 2000).

En Cándido encontramos el dispositivo de personalización, es decir que, a través de su representación, las autoras esperaban que el lector se identificara, 
se encontrara cercano a sus expresiones, actitudes y vestuario. ${ }^{12} \mathrm{Y}$ por tanto, que lo encuentre próximo y que sienta su discurso comprensible. Esto se refuerza con el uso de formas coloquiales y rimas por parte del campesino en el momento de expresarse:

Yo soy Cándido Buendía, campesino de pura cepa y corazón, desde que mis taitas me criaron he arado la tierra; el monte es mi vida y la de mi región, conozco las matas de por aquí, aunque muchas de ellas ya no estén ahí.

Hoy vengo a contarles un poquito de mi saber, pa'que sus hijos dentro de unos años también las puedan conocer, esta cartilla es pa'aprender, sale de mi memoria pa' sumercé. (JBJCM, 2007 p. 9)

Llama la atención que el uso de estas formas de decir, aparecen en el ejemplo y en el material de manera paralela, pero subordinada al uso del nombre científico de la planta y la intencionalidad de describir en detalle la misma. Estas formas de decir de Cándido son lo que hemos denominado marcas de informalidad, en las que predomina el registro coloquial, principalmente presente en las expresiones del campesino protagonista. Estas formas se contraponen, son consustanciales a las marcas de formalidad que se caracterizan por el uso de términos y nociones más específicas, como en las descripciones hechas por la voz del experto:

UCHUVA, PEPINO DULCE, LULO DE PÁRAMO, AJÍ DE CLIMA FRÍO y PAPA BANDERA. Familia: Solanaceae ESPECIES: Physalis peruviana (Uchuva), Solanum muricatum (Pepino dulce, llorón), Solanum phureja (Papa bandera), Solanum quitoense var. septentrionale (Lulo de páramo), Capsicum pubescens (Rocoto, ají de clima frío, siete caldos) (JBJCM, 2007 p. 17).

La condición consustancial, pero diferenciada, de estas dos marcas nos permite identificar la existencia de las dos voces, pero también permite identificar la subordinación de una de ellas, la que se identifica como coloquial y de Bogotá.

${ }^{12}$ Recordemos que la cartilla está dirigida a un público campesino de las zonas periurbanas 
popular a la de aquel que es invisible, "el estudioso" al que se refiere Cándido al inicio de la cartilla.

Esta tensión y consecuente subordinación redunda en las definiciones que se utilizan en la cartilla. Mientras las del experto, ese estudioso invisible, son de carácter técnico, específico, que no son puestas en cuestión y se asumen como certeras. ${ }^{13}$ Las definiciones que hace Cándido, se presentan a través de un lenguaje informal, intentando relacionar las definiciones que da el experto con referentes más cercanos, a partir de versos o coplas que recita. Veamos esto en la imagen 3:

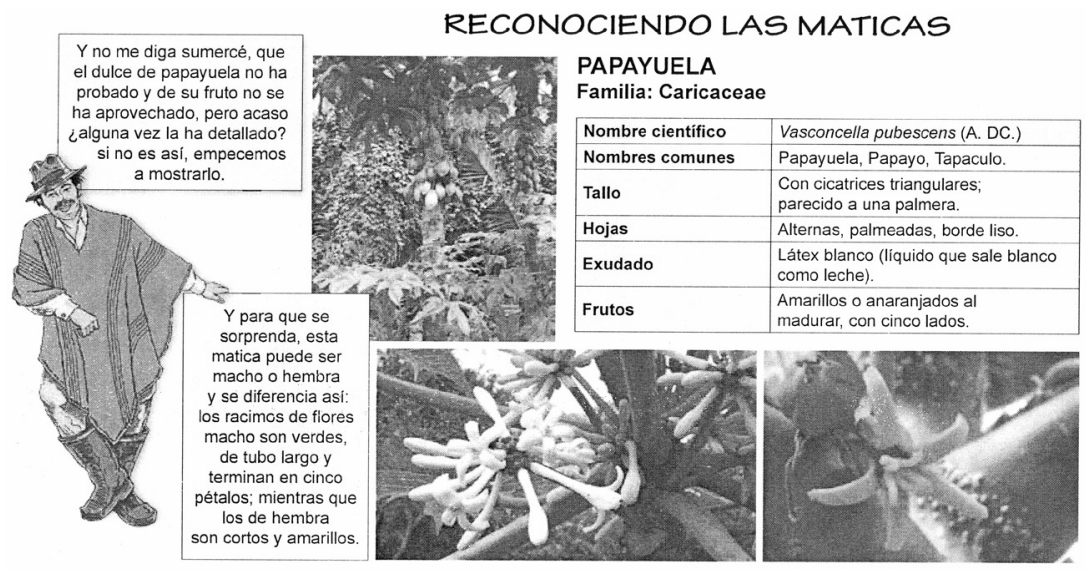

Imagen 3 - Fonte: Cartilla Las Maticas de mi Huerta. Jardín Botánico José Celestino Mutis, Bogotá.

Frente a la papayuela, la voz del experto, describe el nombre científico y las partes de la planta "Hojas: alternas, palmeadas, borde liso" (JBJCM, 2007 p. 10), esta información es validada: todas las hojas de esta planta son iguales, y según parece es fundamental su conocimiento. En tanto, la voz del campesino dice "esta matica puede ser macho o hembra", el uso del diminutivo intenta aligerar la información que se da y la expresión "puede ser" da cuenta de una

${ }^{13}$ Básicamente se concentran en la taxonomía linneana de las plantas, esto se debe a que el material, finalmente, es producido por el Jardín Botánico de Bogotá, de ahí el interés por presentar clasificaciones y órdenes de las plantas. Es en los jardines botánicos, donde se concentran en un espacio reducido grandes cantidades de especímenes naturales y pueden compararse con otros organismos de lugares alejados, ello implica el diseño de formas de representación, sistemas universales de códigos y de reglas, así la taxonomía se convierte en ese método universalizado que permite el orden, la comparación y la representación de la naturaleza en un mapa global. (NIETO, 2000). 
afirmación menos certera que la del experto. Gráficamente, esto se complementa con el uso de una tabla, de un lado, y de un recuadro del otro. La tabla presenta una descripción, no muy minuciosa, pero sí especializada, que incluye términos complejos, nombres técnicos y ejemplificaciones. Estas gráficas parecen estar orientadas a alfabetizar al público objetivo, dada su presentación muy similar a la de un texto escolar (UNESCO, 1994). No hay justificación del porqué deben comprenderse estas clasificaciones, pero dada la recurrencia de este tipo de gráficos en la cartilla, es posible afirmar que quienes la diseñaron comprendían que era fundamental que esta información se explicara. De otro lado, el recuadro en el que se expone la explicación del campesino, separa, distancia, señala la diferencia entre el discurso técnico (asociado a gráficos y tablas), del discurso divulgativo (asociado a lo literario con el uso de esta viñeta propia de las caricaturas y los comics).

En la imagen 4 podemos ver cómo en la viñeta se narrativiza la voz de Cándido a través de recursos como rimas "Unas ricas papas bandera lleniticas de ají y para completar una sabrosa ensalada de uchuva y llorón, dos frutos que bien sazonados saben mejor". Y se presenta su figura escenificando una situación familiar. En tanto, las fotos ejemplifican, intentando reproducir lo más fielmente posible la realidad, debajo de las mismas se encuentran sus nombres científicos, sin apelar a ningún tipo de metáfora u otro recurso literario. La ilustración no dialoga con las fotografías, son imágenes separadas, donde parece más fiel y menos caricaturezca la voz del experto.

A través de los dispositivos del lenguaje y gráficos pudimos encontrar que se presenta un contenido como accesorio, que se instrumentaliza para intentar
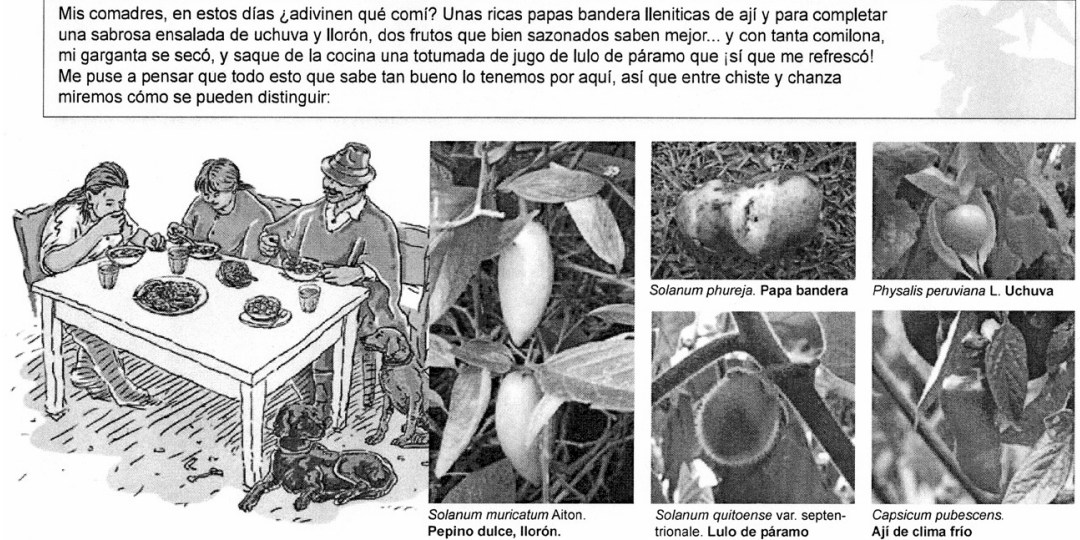

Imagen 4 - Fonte: Cartilla Las Maticas de mi Huerta. Jardín Botánico José Celestino Mutis, Bogotá. 
llegar a un público específico, pero que entre más figura en el material, más se revela su lugar de subordinación. Ello se relaciona con un modelo de déficit en tanto que se jerarquiza el conocimiento, privilegiando un tipo de ciencia y tecnología, sobre el saber popular. Las expresiones que apelan a conocimientos tradicionales se usan instrumental y accesoriamente.

Esta asociación de la popularización con lo banal e instrumental está acompañada - se lee a dúo - con un posicionamiento subordinado de esta práctica a una producción de conocimiento hegemónica; subordinación que se traduce en la reproducción desde mecanismos estandarizados de un ideal de ciencia y tecnología establecida y dominante (PÉREZ-BUSTOS, 2010a, 2011).

\section{Consideraciones finales}

En el artículo buscamos dilucidar la forma en que se materializa el modelo deficitario entre ciencia y público, que es evidenciado por otros estudios sobre ASCyT en el país, en el caso de la cartilla Las Maticas de mi Huerta, que como lo mencionamos, está dirigida a una población campesina. Ubicamos esta exploración en un contexto socio-histórico que enmarca la producción de estas materialidades edu-comunicativas caracterizado por una subordinación de la actividad de los mediadores y de los públicos. El análisis se centró en identificar y describir los dispositivos de lenguaje y diagramación de la cartilla en los que dicho modelo se soporta.

A través de estos dispositivos pudimos encontrar que este modelo presenta un conocimiento jerarquizado que privilegia un tipo de ciencia, sobre un saber popular. Y esto nos permitió evidenciar que de un lado, se legitima la voz del experto, y del otro, se utiliza como accesoria la voz del campesino. Estos resultados nos llevan a preguntarnos si son posibles en nuestro contexto, otras formas de comunicar el conocimiento científico y a plantear la necesidad de promover la formación crítica sobre y desde el quehacer de quienes producen estas materialidades edu-comunicativas.

Con estas consideraciones proponemos algunas preguntas como punto de partida para una futura reflexión sobre la producción de estos materiales: ¿cómo presentar el conocimiento científico cuestionando la idea de que es universal y neutro, hecho desde "ninguna parte"?, ¿cómo construir recursos gráficos y de lenguaje que rompan la dicotomía entre la voz invisible del experto y la de los públicos?, ¿cómo representar a expertos y públicos sin caer en estereotipos y caricaturizaciones? 
Igualmente, los resultados encontrados sugieren preguntas en torno a las condiciones en las que se producen estos materiales, y también en torno a cómo son interpretados estos contenidos por los públicos a los que están dirigidos. Como mencionábamos en la introducción, esto hace parte de la investigación más amplia con las autoras de este material y con otros mediadores que está actualmente en desarrollo.

\section{REFERENCIAS}

ALTHEIDE, D. Ethnographic Content Analysis. Qualitative Sociology, v. 10, n. 1, p. 65-77, mar. 1987.

BERRUECOS, L. Las dos caras de la Ciencia: representaciones sociales en el discurso. Revista Iberoamericana de Discurso y Sociedad, v. 2, n. 2, p. 105 -130, jun. 2005.

CALSAMIGLIA, H.; TUSÓN, D. La textura discursiva. In: Las cosas del decir. Barcelona: Ariel, 1999. p. 217-250.

; BONILLA, S.; CASSANY, D.; LÓPEZ, C.; MARTÍ, J. Análisis discursivo de la divulgación científica. Lengua, discurso y texto, v. 2, p. 639-646, jul. 2001.

CASSIANI, S.; LINSINGEN, I.; GIRALDI, P. Histórias de leituras: produzindo sentidos sobre ciência e tecnologia. Pro-Posições, Campinas, v. 22, n. 1, p. 59-70, 2011.

CASTRO-GÓMEZ, S. La postcolonialidad explicada a los niños. Popayán, 2005. Disponible en: $<$ http://www.cholonautas.edu.pe/modulo/upload/La\%20postcolonialidad $\% 20$ explicada\%20a\%20los\%20ni\%F1os.pdf $>$. Acceso en: 05/11/2011.

DAZA, S.; ARBOLEDA, T. Comunicación Pública de la Ciencia en Colombia: ¿Políticas para la democratización del conocimiento? Signo y Pensamiento, v. 26, n. 50, p. 101-125, ene.-jun. 2007.

FRANCO AVELLANEDA, M.; PÉREZ-BUSTOS, T. ¿De qué ciencias hablan nuestros materiales de divulgación? Revista Colombiana de Educación, Bogotá, n. 56, p. 78, 2009.

Tensiones y convergencias entorno a la Apropiación Social de la Ciencia y la Tecnología en Colombia. In: PÉREZ-BUSTOS T.; TAFUR SEQUERA, M. (Eds.). Deslocalizando la apropiación social de la ciencia y la tecnología: Aportes desde Prácticas Diversas. Bogotá, Colombia: Maloka-Colciencias, p. 9, 2010.

GALIETA NASCIMENTO, T.; CASSIANI, S. Leituras de divulgação científica por licenciandos em Ciências Biológicas. Revista Electrónica de Enseñanza de las Ciencias, Bogotá, v. 8, n. 3, 2009. 
HALL, S. Representation: cultural representations and signifying practices. London: Sage. Londres, 1997.

JARDÍN BOTÁNICO JOSÉ CELESTINO MUTIS. Las maticas de mi huerta. Bogotá: Jardín Botánico José Celestino Mutis y Secretaría de Ambiente, 2007.

KANT, I. Respuesta a la pregunta ¿qué es la ilustración? Berlín, 1783.

NIETO, M. Remedios para el Imperio: Historia Natural y apropiación del Nuevo Mundo, Bogotá: ICANH, 2000.

ORLANDI, E. Análise de discurso. Principios e procedimentos. Campinas: Ponte Editores, 2009.

PÉREZ-BUSTOS, T. La feminización cultural de las prácticas educativas: etnografías de la popularización de la ciencia y de la tecnología en dos países del sur. Revista CS, Cali, v. 6, n. 17, p. 77-103, 2010a.

. Apuntes feministas para situar la popularización de la ciencia en el sur global. In: VESSURI, H.; KREIMER, P.; ARELLANO, A.; SANZ MENÉNDEZ, L. (Eds.). Conocer para transformar: producción y reflexión sobre Ciencia, Tecnología e innovación en Iberoamérica. Caracas: UNESCO-IESALC, 2010b, p. 275-293.

. Aportes feministas a la Educación popular: entradas para repensar pedagógicamente la popularización de la ciencia y la tecnología. Educação e Pesquisa, São Paulo, v. 36, n. 1, p. 243-60, 2010c.

. Feminización y popularización de ciencia y tecnología en la política científica colombiana e india. Revista Iberoamericana de Ciencia, Tecnología y Sociedad, Buenos Aires, v. 6, n. 17, p. 77-103, 2011.

; FRANCO AVELLANEDA, M.; LOZANO BORDA, M.; FALLA, S.; PAPAGAYO, D. Iniciativas de apropiación social de la Ciencia y la Tecnología en Colombia: Tendencias y retos para una comprensión más amplia de estas dinámicas. Revista História, Ciências e Saúde, Manguinhos, Rio de Janeiro, v. 19, n. 1, p. 115-137, 2012.

UNESCO. Módulo para la edición de textos escolares. Bogotá: Cerlalc, 1994.

Texto recebido em 09 de novembro de 2011.

Texto aprovado em 06 de dezembro de 2011. 